\title{
A shortcoming and deficiency in "the prevalence of knee injuries ipsilateral to tibial shaft fractures and their impact on clinical outcome"
}

\author{
Yueju Liu $\cdot$ Yingze Zhang
}

Received: 5 May 2014/ Accepted: 6 May 2014/Published online: 16 May 2014

(C) Springer-Verlag France 2014

Dear editor,

We read with interest the study of prevalence of knee injuries ipsilateral to tibial shaft fractures and their impact on clinical outcome [1] and wish to congratulate the authors on their excellent work. However, we have found there was a shortcoming and deficiency regarding the paper and wish to share them.

Firstly, the age of patients in this study ranged from 18 to 62 years, which spanned from youth until old age. However, tibial shaft fractures can be divided into two high-energy and low-energy trauma injuries. The highenergy injury is common in young patients, and often accompanied with knee injury, especially in open fracture and comminuted fractures of the upper tibial shaft. This has been confirmed by previous study [2] in 1981 and accepted by most orthopedic trauma doctors. While the low-energy injury is common in elderly patients, which often accompanied osteoporosis, whose tibial fracture can be occurred in simple fall or minor traffic accident. Such kind of tibial shaft fracture is typically oblique fracture, most of whom do not have knee injuries. However, a considerable of such patients have meniscal tears or intra-meniscal signal abnormalities long years ago, which are very easily lead to disputes in a road accident. Therefore, this study includes both young and elderly patients is very unscientific. It may

\section{Y. Liu · Y. Zhang $(\bowtie)$}

Department of Orthopedic Center, Third Hospital of Hebei

Medical University, No. 139 Zi Qiang Road,

Shijiazhuang 050051, Hebei, People's Republic of China

e-mail: yzlingliu@126.com

Y. Liu · Y. Zhang

Key Orthopaedic Biomechanics Laboratory of Hebei Province,

Shijiazhuang, People's Republic of China

e-mail: liuyueju1983@gmail.com underestimate the incidence of knee injury in young patients with tibial shaft fractures.

In our limited experience for nearly 80 comminuted tibial shaft fractures in the young adults, incidence of knee injury is as high as $98.6 \%$. And nearly $8.2 \%$ of those needed surgical treatment of meniscus and anterior cruciate ligament rupture. So we think that routine use of preoperative knee MRI examination in such young patients with tibial shaft fractures is necessary, if the clinical examination to seek suspicious knee injury under general anesthesia just after the fracture fixation.

Conflict of interest The authors confirm that there is no known conflict of interest associated with this publication and there has been no significant financial support for this work that could have influenced its outcome.

\section{References}

1. Guler F, Kose O, Erol B, Turan A, Koroglu M, Akalin S (2014) The prevalence of knee injuries ipsilateral to tibial shaft fractures and their impact on clinical outcome. Eur J Orthop Surg Traumatol. doi:10.1007/s00590-014-1465-5

2. Muckle DS (1981) The unstable knee: a sequel to tibial fractures. In: Proceedings of the British Orthopaedic Association. J Bone Joint Surg Br 63B: 628 